\title{
Irrigantes usados en dientes deciduos y su impacto citotóxico en fibroblastos de ratón
}

Irrigants used in temporary teeth and their cytotoxic impact on mouse fibroblasts
Irrigantes usados em dentes temporários e seu impacto citotóxico em fibroblastos de camundongos
Fecha de Recepción

23 de mayo 2019
Aceptado para su publicación

23 de junio 2019
Daniela Yanina Burlli

Asignatura Odontopediatría, Módulo Atención Integral del Niño y Adolescente. Facultad de Odontología. Universidad Nacional del Nordeste.

María Lorena Cardoso Asignatura Odontopediatría, Módulo Atención Integral del Niño y Adolescente. Facultad de Odontología. Universidad Nacional del Nordeste.

Juan Santiago Todaro Asignatura Bioquímica. Facultad de Medicina. Universidad Nacional del Nordeste.

Tamara Barnes

Asignatura Bioquímica. Facultad de Medicina. Universidad Nacional del Nordeste.

Juan Pablo Melana Colavita Asignatura Bioquímica. Facultad de Medicina. Universidad Nacional del Nordeste.

\section{Autor de Correspondencia}

Daniela Yanina Burlli

Facultad de Odontología. UNNE. Av. Libertad 5450. Corrientes (Capital). República Argentina. Email: danielayburlli@gmail.com

\section{Resumen}

La atención odontopediátrica se encuentra limitada a tiempos de ejecución cortos necesitando mayor dependencia a agentes antimicrobianos de rápida eficacia para eliminar y prevenir cualquier desarrollo de microorganismos. Un gran número de irrigantes químicos son utilizados para la limpieza de los conductos radiculares. El objetivo del presente trabajo fue evaluar la citotoxicidad de irrigantes utilizados para la irrigación de conductos en piezas dentarias primarias y determinar la viabilidad, in vitro, en cultivo de fibroblastos de ratón (L-929), mediante el bioensayo de colorimetría rápida de MTT, a diferentes tiempos de incubación. Los irrigantes ensayados fueron: Hidróxido de Calcio $\left(\mathrm{Ca}(\mathrm{OH})_{2}\right)$, Hipoclorito de sodio $(\mathrm{NaClO})$ y Fluoruro de Diamino Plata (FDP). La viabilidad de los fibroblastos a las drogas irrigantes se evaluó con diferentes dosis de los mismos y a diferentes tiempos, en función de la adherencia celular. Los ensayos fueron llevados a cabo por triplicado. Las células fueron fotografiadas y contabilizadas bajo microscopio óptico invertido. Los datos obtenidos, fueron analizados y graficados en manera descriptiva. Estos resultados mostraron que las células del grupo control se mantuvieron viables y sin modificaciones pertinentes. No obstante, se observó citotoxicidad celular y reducción de la viabilidad con los diferentes tratamientos dependiendo de las dosis y tiempos de exposición a los mismos. Cuando los fibroblastos se incubaron, durante 10 minutos, con las diferentes drogas $\mathrm{Ca}(\mathrm{OH})_{2}$, 
FDP y $\mathrm{NaClO}$ ) y disoluciones, la viabilidad se vio afectada a la mayor concentración de cada droga; en la mayor concentración de $\mathrm{NaClO}$ y $\mathrm{Ca}$ $(\mathrm{OH})_{2}$ se produjeron los mayores porcentajes de alteración celular, sin embargo, solo con el $\mathrm{NaClO}$, hasta en las mínimas diluciones se registraron viabilidades inferiores a las registradas en el control.

Palabras Claves: Citotoxicidad, Irrigantes del Conducto Radicular, Hipoclorito de Sodio, Hidróxido de Calcio, Fluoruro Diamino de Plata.

\section{Abstract}

Odontopediatric care is limited to short execution times. It requires greater dependence on antimicrobial agents of rapid efficacy to eliminate and prevent any development of microorganisms. A large number of chemical irrigants are used to clean the root canals. The aim of the present work was to evaluate the cytotoxicity of irrigants used for the irrigation of the root canals in primary dental pieces and to determine the viability, in vitro, in culture of mouse fibroblasts (L-929), by means of the rapid MTT colorimetry bioassay, at different incubation times. The irrigants tested were: Calcium Hydroxide $\mathrm{Ca}(\mathrm{OH})_{2}$, Sodium Hypochlorite $(\mathrm{NaClO})$ and Diamine Silver Fluoride (FDP). The viability of fibroblasts to irrigating drugs was evaluated with different doses of them and at different times, depending on cell adhesion. The tests were carried out in triplicate. The cells were photographed and counted under an inverted optical microscope. The data obtained was analyzed and graphed in a descriptive way. These results showed that the cells of the control group remained viable and without relevant modifications. Nevertheless, cellular cytotoxicity and reduction of viability were observed with the different treatments depending on the doses and times of exposure to them. When the fibroblasts were incubated, for 10 minutes, with the different drugs $\mathrm{Ca}(\mathrm{OH})_{2}, \mathrm{FDP}$ and $\mathrm{NaClO}$ ) and solutions, the viability was affected at the highest concentration of each drug; in the highest concentration of $\mathrm{NaClO}$ and $\mathrm{Ca}(\mathrm{OH})_{2}$, the highest percentages of cellular alteration were produced, however only with $\mathrm{NaClO}$, even in the lowest dilutions there were lower viabilities than those recorded in the control.

Key Words: Cytotoxicity, Irrigating the Root Canal, Sodium Hypochlorite, Calcium Hydroxide, Diamino Fluoride Silver.

\section{Resumo}

atendimento odontopediátrico é limitado a tempos de execução curtos, exigindo maior dependência de agentes antimicrobianos de eficácia rápida para eliminar e prevenir qualquer desenvolvimento de microorganismos. Um grande número de irrigantes químicos é usado para limpar os canais radiculares. $O$ objetivo do presente trabalho foi determinar a citotoxicidade de irrigantes utilizados na irrigação de ductos em peças primárias odontológicas e determinar a viabilidade, in vitro, da cultura de fibroblastos de camundongos (L-929), por meio da colorimetria rápida de MTT. bioensaio, em diferentes tempos de incubação. Os irrigantes testados foram: Hidróxido de Cálcio $\mathrm{Ca}(\mathrm{OH})_{2}$, Hipoclorito de Sódio $(\mathrm{NaClO})$ e Diamine Silver Fluoride (FDP). A viabilidade de fibroblastos para irrigar drogas foi avaliada com diferentes doses e em diferentes tempos, dependendo da adesão celular. Os testes foram realizados em triplicado. As células foram fotografadas e contadas sob um microscópio óptico invertido. Os dados obtidos foram analisados e representados graficamente de forma descritiva. Esses resultados mostraram que as células do grupo controle permaneceram viáveis e sem modificações relevantes. Entretanto, a citotoxicidade celular e a redução da viabilidade foram observadas com os diferentes tratamentos, dependendo das doses e tempos de exposição a elas. Quando os fibroblastos foram incubados, por 10 minutos, com os diferentes fármacos $\mathrm{Ca}(\mathrm{OH})_{2}$, FDP e $\left.\mathrm{NaClO}\right)$ e soluções, a viabilidade foi afetada na maior concentração de cada fármaco; na maior concentração de $\mathrm{NaClO}$ e $\mathrm{Ca}(\mathrm{OH})_{2}$, os maiores percentuais de alteração celular foram produzidos, porém apenas com $\mathrm{NaClO}$, mesmo nas menores diluições foram menores as viabilidades que as registradas no controle. 
REVISTA FACULTAD DE ODONTOLOGIA ISSN 1668-7280 / ISSN-E 2683-7986

Palavras chave: Citotoxicidade, Irrigantes do Canal Radicular, Hipoclorito de Sódio; Hidróxido de Cálcio; Diamino Fluoreto de Prata.

\section{Introducción}

En la práctica odontológica se utilizan numerosas sustancias para el tratamiento de las piezas dentarias afectadas, que ejercen efecto localmente y por difusión hacia los tejidos circundantes. Teniendo la Odontopediatría, como objetivo principal la conservación de las piezas dentarias temporarias en el arco hasta su normal época de recambio, el profesional lleva a cabo diversos tratamientos utilizando una gran variedad de materiales y fármacos, a fin de rehabilitar las piezas dentarias temporarias que presenten alteraciones infecciosas o traumáticas'.

La atención odontopediátrica, se encuentra limitada a tiempos de ejecución cortos, generando una mayor dependencia a agentes antimicrobianos que posean eficacia para eliminar y prevenir cualquier crecimiento de microorganismos. Una gran cantidad de productos químicos son utilizados para la limpieza de conductos radiculares, incluyendo $\mathrm{Ca}(\mathrm{OH})_{2},{ }^{2} \mathrm{NaClO}^{3,4}$ y FDP5. $\mathrm{La}$ biocompatibilidad de los materiales es de suma importancia, sobre todo cuando se emplean en niños donde las concentraciones toxicas y de difusión son extremas.

Remitiéndonos a la literatura en odontopediatría, el uso de topicaciones con FDP al $38 \%$ es habitual en la práctica diaria, como cariostático y remineralizante. De esta manera, se procede al control de la caries en forma no traumática, factor importante para el tratamiento en un niño ${ }^{6,7}$. Otra de las propiedades del FDP es que tiene mayor penetración en comparación con otros fluoruros tópicos. Por las propiedades descriptas FDP es un excelente cariostático, remineralizante y bactericida del tejido desorganizado ${ }^{8-10}$.

Otro de los irrigantes seleccionados es el $\mathrm{Ca}(\mathrm{OH})_{2}$, el cual es un medicamento intraconducto que posee un $\mathrm{pH}$ alcalino de aproximadamente 12.5, lo que le confiere actividad bactericida mediante la liberación de iones hidroxilos que se introducen en la membrana citoplasmática bacteriana provocando hidrólisis de moléculas e inhibiendo actividades enzimáticas bacterianas. Así mismo, el $\mathrm{Ca}(\mathrm{OH})_{2}$, posee capacidad para disolver el tejido pulpar y para activar a la fosfatasa alcalina, necesaria para la iniciación del proceso de mineralización".

El $\mathrm{NaClO}$, es una sal formada por la unión de dos compuestos químicos, el ácido hipocloroso y el hidróxido de sodio, que presenta como características principales sus propiedades oxidantes. Los beneficios que proporciona el $\mathrm{NaClO}$ como irrigante durante la terapia endodóntica son: efectividad para eliminar el tejido vital y no vital, con un amplio efecto antibacteriano, destruyendo bacterias, hongos, esporas y virus; es excelente lubricante $y$ blanqueador, favoreciendo la acción de los instrumentos, posee una tensión superficial baja, vida media de almacenamiento prolongada, $y$ es poco costoso ${ }^{12}$.

El uso clínico de estas soluciones ha demostrado su eficacia y seguridad así como su potencia al mantener los tejidos libres de infección. Además, se ha sugerido que algunas de éstas pueden tener un efecto sinérgico, relacionado con la modulación de procesos inflamatorios y de cicatrización ${ }^{13}$.

El objetivo del presente trabajo fue evaluar la citotoxicidad de irrigantes utilizados en la actualidad, empleados para la irrigación de conductos en piezas dentarias primarias y determinar mediante ensayos in vitro en cultivo de fibroblastos de ratón (L-929), a través el bioensayo de colorimetría rápida de MTT, a diferentes tiempos de incubación ${ }^{14,15}$.

\section{Materiales y métodos}

\section{Soluciones Irrigantes}

Se prepararon soluciones estériles de los 3 irrigantes más utlizados en la actualidad:

Solución de $\mathrm{Ca}(\mathrm{OH})_{2}$ al: $0,1 \%, 0,5 \%, 1 \%$ y $2 \%$ en Solución fisiológica. Solución de $\mathrm{NaClO}$ al: $0,1 \%$, $0,5 \%, 1 \%$ y $2 \%$ en Solución fisiológica y solución de FDA al: $3,8 \%, 5 \%, 10 \%$ y $38 \%$ en solución fisiológica. Dichas soluciones irrigantes fueron utilizadas para medir su efectividad y efecto citotóxico a partir de una cinética de reacción llevada a cabo a diferentes tiempos: I, 2, 5 y 10 minutos de exposición. 


\section{Cultivos celulares}

Se utilizó la línea de células de fibroblastos de ratón (L-929), adquirida de American Type Culture collection (ATCC). Esta línea celular es un clon estable de la línea fibroblástica murina, similar a las presentes en la pulpa dental humana, siendo actualmente, un modelo de estudio validado y representativo de piezas dentarias primarias en cultivo.

Mantenimiento de los cultivos y Subcultivos La línea celular se mantuvo en Dulbecco's minimum essential medium (DMEM) (GIBCOInvitrogen) con el agregado de 10\% de suero fetal bovino (SFB) inactivado por calor (GIBCOInvitrogen), L-Glutamina $(29,2 \mathrm{mg} / \mathrm{mL})$, Penicilina ( 10,000 unidades $/ \mathrm{mL})$ y Estreptomicina $(10,000$ $\mu \mathrm{g} / \mathrm{mL}$ ) (GIBCO-Invitrogen) como antibióticos (Fogh, 1978). Cuando las células Ilegaron a condiciones de confluencia, se procedió a subcultivar para asegurar el crecimiento exponencial de las mismas. El subcultivo celular, permitió controlar la densidad del inóculo inicial que asegura que se trabajará en condiciones de crecimiento óptimas. Para ello, se observó al microscopio la botella que se subcultivó para ver si la monocapa es continua y uniforme, se eliminó el medio de cultivo y se lavó con PBS sin $\mathrm{Ca}++$ ni $\mathrm{Mg}++$. Se cubrió la monocapa con cantidad suficiente de una solución de Tripsina-EDTA 0.25\% (GIBCOInvitrogen) y se incubó durante 3-5 minutos a $37^{\circ} \mathrm{C}$. Una vez que la monocapa se despegó, se agregó suero fetal bovino para inactivar la enzima, realizándose sucesivos pasos de pipetas para disgregar las células. Se centrifugó a 800 - 1000 rpm durante 5 a 10 minutos, descartándose el sobrenadante y disolviéndose el pellet en medio suplementado con suero. Luego del recuento celular con azul de tripan, entre 0,5 - I . I05 cél/ $\mathrm{mL}$ se sembraron en placas o frascos estériles y se incubaron en estufa de cultivos a $37{ }^{\circ} \mathrm{C}$ y $5 \%$ de $\mathrm{CO}_{2}$.

\section{Ensayos de citotoxidad y proliferación ce- Iular'6}

Se utilizaron células obtenidas de monocapas subconfluentes. Las células resuspendidas se sembraron en placas de 96 pocillos, I.5-2.5 × $10^{4}$ células por pocillo, en el mismo medio de crecimiento (DMEM-SFB I0\%). Al alcanzar la mono- capa un $60 \%$ de confluencia, se retiró el medio de cultivo y se adicionaron los antagonoistas y/o condicionantes del crecimiento celular a evaluar. Luego de un tiempo de incubación a $37^{\circ} \mathrm{C}$ y $5 \%$ de $\mathrm{CO}_{2}$ en atmósfera húmeda, que se correspondió con la cinética de cada caso, la viabilidad celular fue cuantificada usando CellTiter 96® Non-Radioactive Cell Proliferation Assay (MTT) (Promega), según instrucciones del fabricante. Los experimentos se realizaron por triplicado en al menos tres ensayos independientes, siendo validados mediante conteo celular.

\section{Análisis de la Adherencia}

Se analizó cualitativamente las alteraciones morfológicas y daños celulares inducidos por las diferentes estrategias experimentales propuestas que permitieron correlacionar la expresión y funcionalidad de las células con los parámetros en estudio. Para ello se utilizó un Microscopio invertido Olympus IX5I y se tomaron fotografías con cámara digital Nikon I 3.5 megapíxeles. Para la documentación digital se emplearon distintas aplicaciones en entorno Apple OS X Yosemite.

\section{Análisis Estadístico}

Los datos se expresan en forma descriptiva porcentual, confeccionándose tablas Excel para su registro.

\section{Resultados}

Para determinar los efectos citotóxicos del $\mathrm{Ca}(\mathrm{OH})_{2}$, FDP y $\mathrm{NaClO}$, en fibroblastos de ratón (L-929), se evaluó la viabilidad a través del ensayo de exclusión de Trypan Blue en los diferentes tiempos establecidos. Los resultados obtenidos corresponden a la Media de un mínimo de tres ensayos diferentes realizados por triplicado.

En la Figura I, se expresan los datos de viabilidad obtenidos para el grupo control estableciéndose una viabilidad constante a lo largo del tiempo de incubación empleado en este estudio.

En la Tabla I se observa que, la adición del $\mathrm{Ca}(\mathrm{OH})_{2}$, produce una disminución del $50 \%$ de la adherencia con respecto al control a partir 
de los 10 minutos y en las mayores concentraciones estudiadas $(0,5 \%, 1 \%$ y $2,5 \%)$.

Con el FDP, las diluciones que se utilizaron fueron diferentes a las otras dos drogas, sin embargo, la adherencia celular se afectó en todos los tiempos y concentraciones de forma similar. Los valores de viabilidad no difirieron en más de $25 \%$ con respecto a los valores del control, (Tabla II).

En el caso del $\mathrm{NaClO}$ la disminución celular se vio que al $0,1 \%$ no se producen cambios significativos en cuanto a la adherencia celular en ninguno de los tiempos observados. La disminución significativa de la viabilidad fue directamente proporcional al aumento de la concentración y del tiempo dando como resultado una destrucción total de la célula con la concentración de 2,5\%, (Tabla III).

\section{Discusión}

La preservación de las piezas dentarias primarias es uno de los objetivos más importantes de la odontopediatría, pretendiendo mantener la armonía, el crecimiento y desarrollo de la longitud del arco dental. Por lo tanto, las terapias pulpares deben garantizar un nivel de calidad máximo a fin de conseguir un éxito clínico. Actualmente no existen acuerdos entre los odontopediatras sobre cuál es el irrigante intraconducto más efectivo contra los patógenos pulpares involucrados en procesos de inflamación irreversible, o piezas dentarias infectadas ${ }^{17}$. Las técnicas de irrigación en pulpectomías efectuadas en piezas primarias, representa un paso importante para la lubricación y la remoción de restos necróticos sueltos y contaminados.

Muchos factores deben ser considerados al seleccionar un agente irrigante para pulpectomías en piezas temporarias, en donde su eficacia clínica cobra una gran importancia. Kaur ${ }^{18}$ propuso una serie de requisitos que debe cumplimentar un irrigante intraconducto ideal: I) amplio espectro antimicrobiano, 2) alta eficacia contra anaerobios y microorganismos facultativos organizados en el biofilm, 3) capacidad de disolver tejido pulpar necrótico, 4) capacidad de inactivar endotoxinas, 5) capacidad de prevenir la formación o de disolver la formación de frotis producto de la instrumentación de las paredes del conducto, y 6) no toxico para el tejido periodontal, de naturaleza no caustica, biocompatible con los tejidos blandos. Estas cualidades de biocompatibilidad deben estar correctamente testeadas para el uso sobre todo en odontopediatría; y los cultivos celulares, son uno de los métodos significativamente viables de evaluar a fin de determinar la toxicidad de estas drogas, simulando los tiempos de trabajo que se emplean en el tratamiento $y$ las dosis que habitualmente se utilizan. En este estudio se manipularon células fibroblásticas de ratón ya que estas células proporcionan un modelo más representativo de la población celular de fibroblastos in vivo ${ }^{19}$. Además, los cambios celulares que pueden resultar de la manipulación del cultivo celular, será menor en los cultivos celulares primarios ${ }^{20}$. En este estudio se observó, a través de la viabilidad celular, que la respuesta a las diferentes drogas testeadas, fueron dependientes de la dosis, incrementándose al disminuir la concentración de todos los medicamentos ${ }^{21}$. Los tiempos de exposición seleccionados se basaron en los utilizados por diferentes autores en estudios clínicos. Con respecto al tiempo de exposición se manifestó una relación inversamente proporcional, a medida que éste aumento en todas las diluciones, la viabilidad celular disminuyó.

Sin embargo, en el tiempo promedio (2') y dosis que habitualmente se utilizan estos irrigantes se obtuvieron resultados similares al control. En el caso del FDP (38\%), se redujo un I5\% la viabilidad celular con respecto al control en tanto que el $\mathrm{Ca}(\mathrm{OH})_{2}(2,5 \%), \mathrm{NaClO}(1 \%)$ la reducción de la viabilidad celular se redujo a valores cercanos a la mitad del observado en el grupo control ( $45 \%$ y $59 \%$ respectivamente). Siendo estas últimas dos sustancias las que se emplean con mayor frecuencia en las prácticas de irrigación en la clínica odontopediátrica nos permite inferir que a esa concentración podemos emplearla con cierto margen de seguridad.

Si bien, el éxito de la terapia de la pulpectomía depende de una adecuada desinfección dentro de los conductos radiculares, es un reto para el odontopediatra seleccionar una droga que dentro de su efectividad clínica para eliminar microorganismos no dañe los tejidos circundantes. A partir de este estudio, se realizarán 


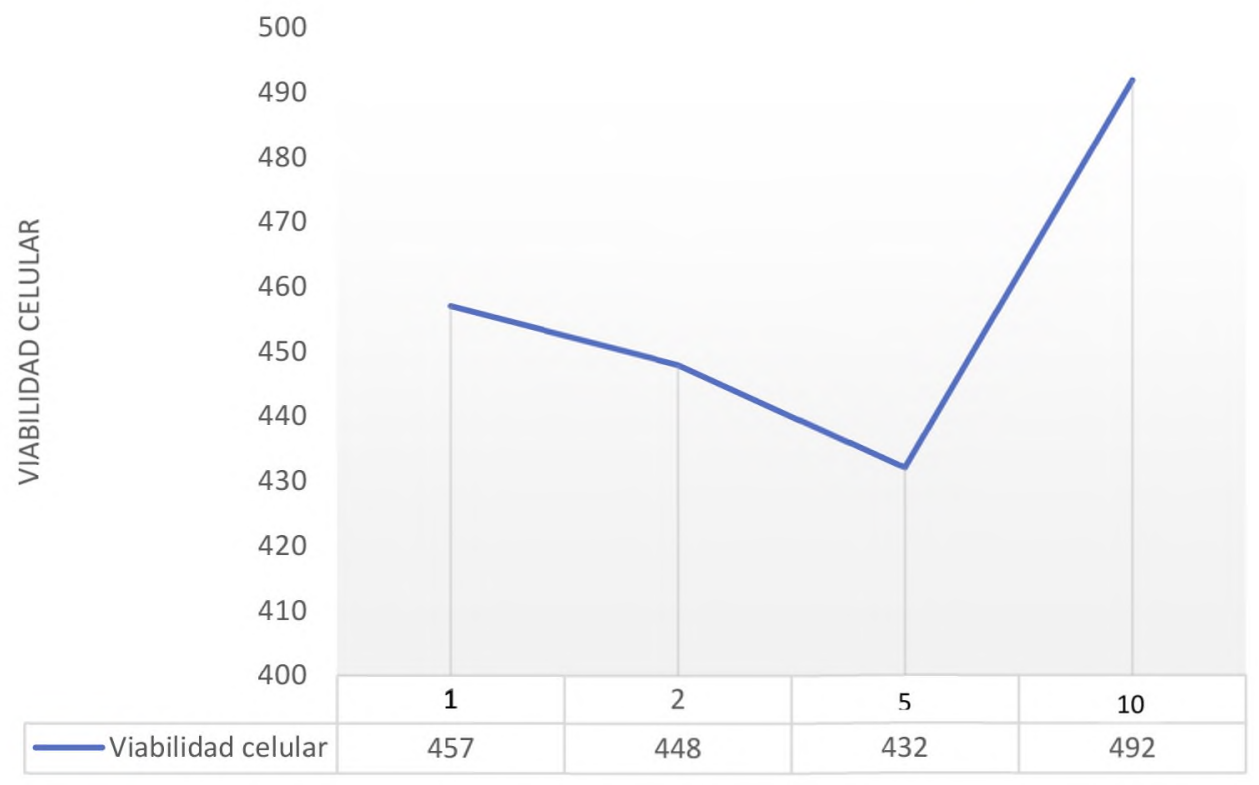

Figura I. Representa el promedio de células contabilizadas en los controles realizados para cada tiempo de incubación prestablecido.

Tabla I. Representa el promedio de células viables, cultivadas con $\mathrm{Ca}(\mathrm{OH})_{2}$, en sus distintas diluciones y contabilizadas en cada tiempo de incubación prestablecido.

\begin{tabular}{|c|c|c|c|c|}
\hline $\mathrm{Ca}(\mathrm{OH})_{2}$ & $\mathrm{I}$ & 2 & 5 & 10 \\
\hline $0,1 \%$ & 422 & 386 & 352 & 328 \\
\hline $0,5 \%$ & 396 & 323 & 256 & 207 \\
\hline $1 \%$ & 386 & 275 & 230 & 195 \\
\hline $2,5 \%$ & 312 & 257 & 189 & 180 \\
\hline
\end{tabular}

Tabla II. Representa el promedio de células viables, cultivadas con FDP, en sus distintas diluciones y contabilizadas en cada tiempo de incubación prestablecido.

\begin{tabular}{|c|c|c|c|c|}
\hline FDP & I & 2 & 5 & 10 \\
\hline $3,8 \%$ & 385 & 356 & 351 & 348 \\
\hline $5 \%$ & 396 & 371 & 353 & 351 \\
\hline $10 \%$ & 387 & 376 & 364 & 369 \\
\hline $38 \%$ & 385 & 381 & 372 & 359 \\
\hline
\end{tabular}

Tabla III. Representa el promedio de células viables, cultivadas con $\mathrm{NaClo}$, en sus distintas diluciones y contabilizadas en cada tiempo de incubación prestablecido.

\begin{tabular}{|c|c|c|c|c|}
\hline $\mathrm{NaClO}$ & $\mathrm{I}$ & 2 & 5 & 10 \\
\hline $0,1 \%$ & 327 & 309 & 301 & 303 \\
\hline $0,5 \%$ & 326 & 252 & 207 & 84 \\
\hline $1 \%$ & 207 & 172 & 61 & 22 \\
\hline $2,5 \%$ & 0 & 0 & 0 & 0 \\
\hline
\end{tabular}


REVISTA FACULTAD DE ODONTOLOGÍA

ISSN 1668-7280 / ISSN-E 2683-7986

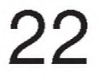

INVESTIGACIÓN

Burlli - Cardoso - Todaro - Barnes - Melana Colavita

http://dx.doi.org/10,30972/ffo,1213870 evaluaciones más precisas para determinar los cambios morfológicos que sufren los fibroblastos al ser incubadas con estas sustancias.

\section{Conclusiones}

- La viabilidad de los fibroblastos en cultivo, se mantuvo constante durante todos los tiempos en los que se realizó el ensayo.

- Cuando se incubaron los fibroblastos con las diferentes drogas $\left(\mathrm{Ca}(\mathrm{OH})_{2}\right.$, FDP y $\left.\mathrm{NaClO}\right)$ y disoluciones durante los 10', la viabilidad se vio afectada a la mayor concentración de la droga.

- A mayor concentración de $\mathrm{NaClO}$ y $\mathrm{Ca}(\mathrm{OH})_{2}$ se produjeron los mayores porcentajes de alteración celular disminuyendo en función a las dosis.

- La destrucción celular total se identificó a partir de la dosis más alta de $\mathrm{NaClO}$ al 2,5\% en todos los tiempos observados.

\section{Referencias Bibliográficas}

I. Hamama HH,Yiu CK, Burrow MF. Effect of silver diamine fluoride and potassium iodide on residual bacterial in dentinal tubules. Aus Dent J. 20I5; 60 (I): 80-87

2. Mei ML, Chu $\mathrm{CH}$, Low $\mathrm{KH}$, Che $\mathrm{CM}$, Lo EC. Caries arresting effect of silver diammine fluoride on dentine carious lesion with $\mathrm{S}$. mutans and $\mathrm{L}$. acidophilus dual-species cariogenic biofilm. Med Oral Patol Oral Cir Bucal 20 13; 18: e824-831.

3. Wang Z, Shen Y, Ma J, Haapasalo M. The effect of detergents on the antibacterial activity of disinfecting solutions in dentin. J Endod 2012; 38 (7): 948-953.

4. Shetty KP, Satish SV, Kilaru K, Ponangi KC, Venumuddala VR, Ratnakar P. Comparative evaluation of the cytotoxicity of $5.25 \%$ sodium hypochlorite, $2 \%$ chlorhexidine and mixture of a tetracycline isomer, an acid and a detergent on human red blood corpuscles: An in-vitro study. Saudi Endod J 2014; 4 (I): I-6.

5. Samaranayake LP. Essential microbiology for dentistry. 4th edn. Edinburgh: Churchill Livingstone Elsevier, 2012.
6. Sachsenmaier S, Peschel A, Ipach I, Kluba T. Antibacterial potency of VAC GranuFoam Silver ${ }^{\circledR}$ Dressing. Injury. 2013;44:1363-1367.

7. Lansdow AB. Silver in health care: Antimicrobial effects and safety in use. Curr Probl Dermatol. 2006; 33: 17-34.

8. Lansdow $A B$, Williams $A$. How safe is silver in wound care? J Wound Care. 2004; 13 (4): $|3|-6$.

9. Yokoyama K, Kimura Y, Matsumoto K, Fujishima A, Miyazaki T. Preventive effect of tooth fracture by pulsed Nd:YAG laser irradiation with diamine silver fluoride solution. J Clin Laser Med Surg. 200 I; 19 (6): 315-8.

10. Yokoyama K, Matsumoto K, Murase J. Permeability of the root canal wall and occlusion of dentinal tubules by $\mathrm{Ag}(\mathrm{NH} 3) 2 \mathrm{~F}$ : $\mathrm{A}$ comparison of combined use with pulsed Nd:YAG laser or iontophoresis. J Clin Laser Med Surg. 2000; I8 (I): 9-I4.

II. Law A, Messer H. An evidence based analysis of the antibacterial effectiveness of intracanal medicaments. J Endod. 2004; 30: 689-94.

12. Hamama H, Yiu C, Burrow M. Current update of chemomechanical caries removal 
methods. Aust Dent J. 20I4; 59 (4): 446-456.

13. Gu XH, Mao CY, Kern M. Effect of different irrigation on smear layer removal after post space prepration. J Endo. 2009; 35 (4): 393-6.

14. Vouzara T, Koulaouzidou E, Ziouti F, Economides $\mathrm{N}$. Combined and independent cytotoxicity of sodium hypochlorite, ethylenediaminetetraacetic acid and chlorhexidine. Int Endod J. 20। 6; 49 (8): 764-73.

15. Botton G, Pires CW, Cadoná FC, Machado AK, Azzolin VF, Cruz IBM, et al. Toxicity of irrigating solutions and pharmacological associations used in pulpectomy of primary teeth. Int Endod J. 20 I6; 49 (8): 746-54.

16. Foersch M, Jacobs C, Wriedt S, Hechtner $M$, Wehrbein $\mathrm{H}$. Effectiveness of maxillary protraction using facemask with or without maxillary expansion: a systematic review and meta-analysis. Clin Oral Investig. 2015; 19 (6): || $8|-| \mid 92$.

I7. Ravinanthanan M, Hegde MN, Shetty V, Kumari S. Cytotoxicity Evaluation of Combination Irrigant Regimens with MTAD on Two Different Cell Lines. Contemp Clin Dent. 20I8; 9 (2): 255-9.
18. Kaur R, Singh R, Sethi K, Garg S, Miglani S. Review article irrigating solutions in Pediatric Dentistry: literature review and update. J Adv Med Dent Sci. 20 14; 2 (2): I04-II5.

19. Al-Haj Ali SN, Al-Jundi SH, Ditto DJ. In-vitro toxicity of grey MTA in comparison to white MTA on human periodontal ligament fibroblasts. Eur Arch Paediatr Dent. 2014; I5 (6): 429-33.

20. Amaral M, Gomes PS, Lopes MA. Cytotoxicity evaluation of nanocrystalline diamond coatings by fibroblast cell cultures. Acta Biomater. 2009; 5 (2): 755-63.

2I. Tulsani SG, Chikkanarasaiah N, Bethur S. An in vivo comparison of antimicrobial efficacy of sodium hypochlorite and Biopure MTADTM against enterococcus faecalis in primary teeth: a qPCR study. J Clin Pediatr Dent. 20I4; 39 (I): 30-34. 